\title{
Discrimination between Coherent and Incoherent Interfaces using STEM Moiré
}

Junji Yamanaka ${ }^{1}$, Daisuke Izumi ${ }^{2}$, Chiaya Yamamoto $^{2}$, Mai Shirakura ${ }^{3}$, Kosuke Hara ${ }^{2}$ and Keisuke Arimoto $^{2}$

${ }^{1}$ University of Yamanashi, Kofu, Yamanashi, Japan, ${ }^{2}$ University of Yamanashi, Japan, ${ }^{3}$ University of Yamanashi, United States

Studies about STEM moiré, which is a moiré between scanning lines of STEM and crystal lattice, are progressing recently [1-4]. Especially it is considered to be very useful to analyze lattice strains in semiconductor materials. However, most of the studies about STEM moiré are based on highly specified TEM, such as Cs-corrected machines. Our group has been focusing on the usage of STEM moiré without using highly specified machines, because we think that may contribute to many researchers in the field of materials sciences [5-7]. In this study, we show experimental results about both coherent and incoherent heterointerfaces of semiconductors, and demonstrate a simulated images of STEM moiré corresponding to those specimens.

A Si and compositionally step-graded SiGe layers were grown onto Si (110) substrate using MBE. We also prepare Ge/Si (100) using MBE. Then we deposited amorphous-carbon, Pt-Pd and tungsten onto the specimens as protection layers for the FIB process. After that, the specimens were fabricated into the foil for the STEM observation using FIB with the acceleration voltage of 40 and $10 \mathrm{kV}$. A field-emission type STEM (FEI Tecnai Osiris) without any Cs correctors was utilized with an acceleration voltage of $200 \mathrm{kV}$. STEM moiré between the specimens' $\{111\}$ planes and the incidental-electron scanning lines were observed. The nominal period of the scanning lines was set to 311 and $632 \mathrm{pm}$ because the period must have been close to a simple integer of the d-spacing of the target crystal planes. $(\mathrm{dSi}(111)=314 \mathrm{pm}$, $\mathrm{dGe}(111)=327 \mathrm{pm})$ Simulated STEM moiré images were derived from the following way: (1) Decide the unit-cell of the specimen, (2) obtain the projection of the unit-cell to the $\{110\}$ plane, (3) draw 2D lattice points of the incidental electron beam such as 2048 by 2048 or 1024 by 1024, (4) put together the above-mentioned two figures, (5) show bright point when the position of atom and that of the electron beam are close, show dark point when those were not close (in the cases of HAADF). For the simulation of the STEM bright field images, reverse images can be used. This is a simple simulation but very useful to interpret the STEM moiré which was taken experimentally.

Figures $1 \mathrm{a}$ and $1 \mathrm{~b}$ are simulated images of STEM moiré, in the cases of incoherent and coherent interfaces, respectively. These images suggests that discontinuous STEM moiré like the Figure 1a means there exists incoherent interface, continuous STEM moiré like Figure $1 \mathrm{~b}$ means coherent interface. Figures $2 \mathrm{a}$ and $2 \mathrm{~b}$ are experimentally-taken STEM bright field images of compositionally step-graded $\mathrm{SiGe} / \mathrm{Si}$ (110) and $\mathrm{Ge} / \mathrm{Si}$ (100), respectively. It can be interpreted that this $\mathrm{SiGe} / \mathrm{Si}$ (110) shown in the Figure 2 a has coherent interfaces, because it shows a continuous moiré across the interfaces and its appearance is similar to the Figure $1 b$ rather than the Figure 1a. On the contrary, Figure $2 b$ is considered to be the result of Ge/Si with the incoherent interface, because it shows a discontinuous moiré across the interface. In summary, STEM moiré is very useful to distinguish coherent and incoherent interfaces. The main advantage of this technique is that highly-specified TEMs are not required and the data is quite easy to understand.

Acknowledgment: The authors thank Mr. K. Sato, Mr. T. Yamada, Dr. T. Arai and Dr. K. Nakagawa for their supplying the specimens. 

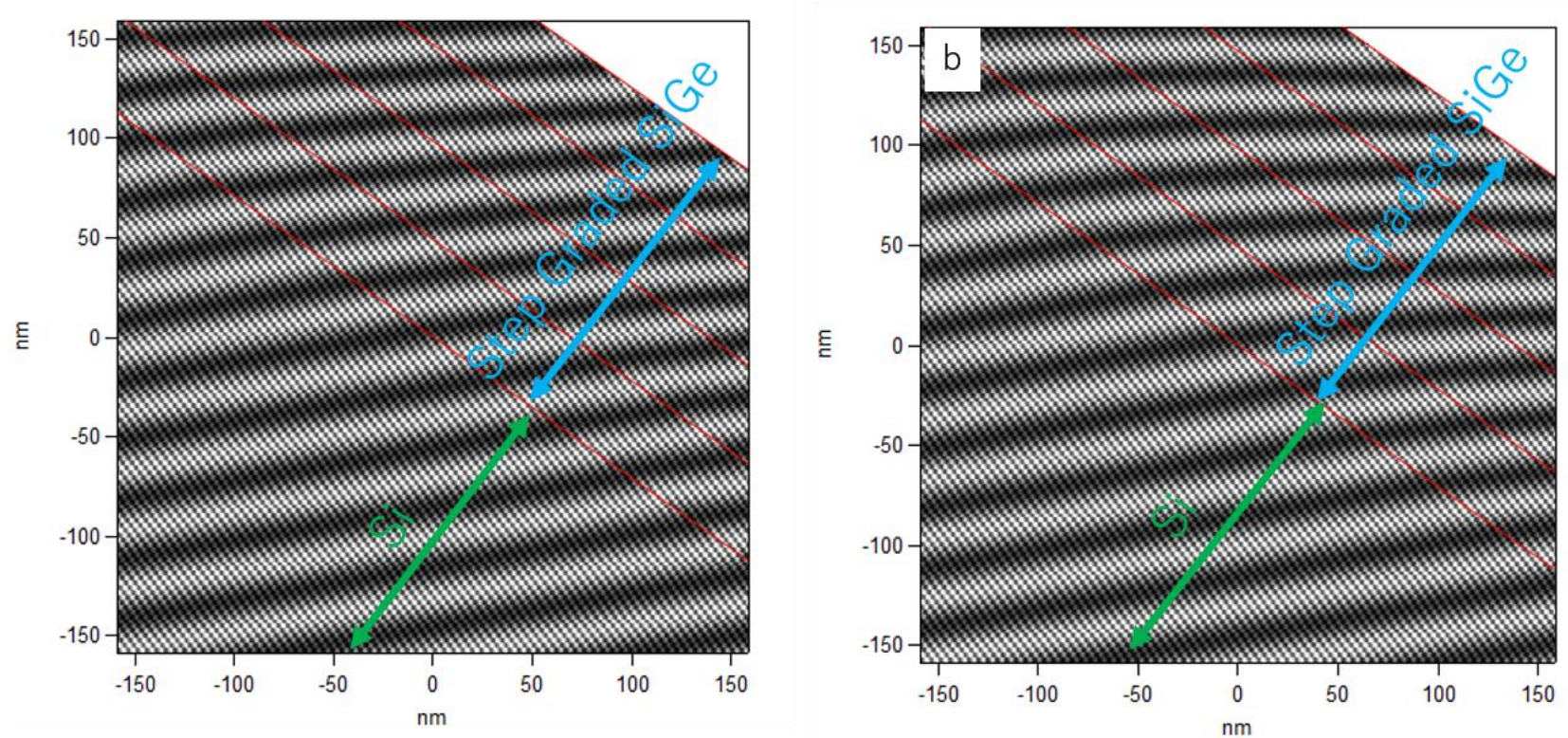

Figure 1. Simulated images of the STEM moiré. The model specimen is the compositionally step-graded $\mathrm{SiGe} / \mathrm{Si}$ (110). The case of incoherent interfaces (a), and that of coherent interfaces (b), respectively.
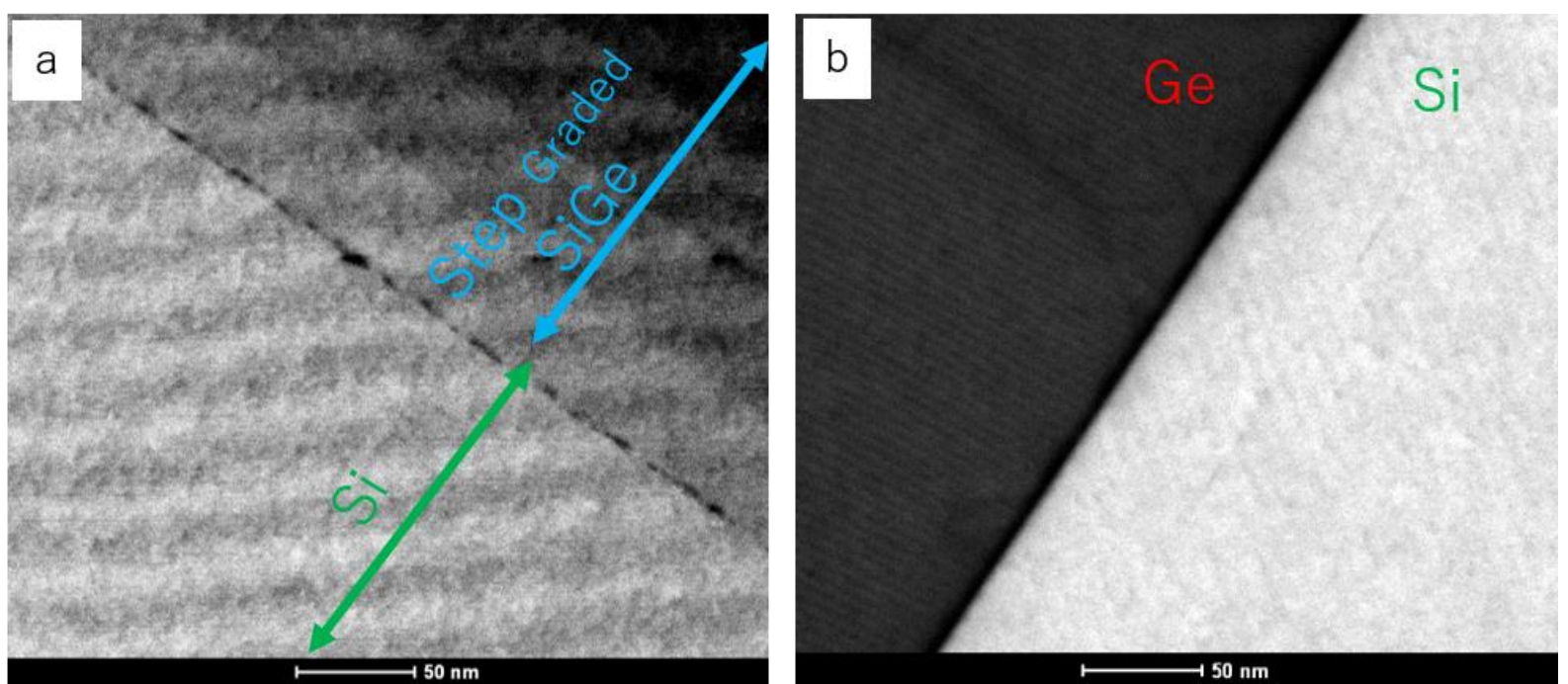

Figure 2. Experimental data of the STEM moiré. (a): A STEM bright field image of the compositionally step-graded $\mathrm{SiGe} / \mathrm{Si}$ (110). The nominal scan period is $311 \mathrm{pm}$. (b): A STEM bright field image of the $\mathrm{Ge} / \mathrm{Si}$ (100). The nominal scan period is $632 \mathrm{pm}$.

\section{References}

[1] S. Kim et al., Appl. Phys. Lett. 102 (2013) 161604.

[2] Y. Kondo and N. Endo, Kenbikyo 49 (2014) 226, in Japanese.

[3] A. Ishizuka et al., J. Electron Microsc. 66 (2017) 217.

[4] A. Pofelski et al., Ultramicroscopy 223 (2021) 113225

[5] Junji Yamanaka et al., J. Mat. Sci. \& Chem. Eng., 5 (2017) 102.

[6] Junji Yamanaka et al., J. Mat. Sci. \& Chem. Eng., 6 (2018) 8.

[7] Junji Yamanaka et al., Microsc. Microanal. 25 (Suppl 2) (2019) 242. 